\title{
On Regularity of a Weak Solution to the Navier-Stokes Equations with the Generalized Navier Slip Boundary Conditions
}

\author{
Jiří Neustupa ${ }^{1}{ }^{1}$ and Patrick Penel ${ }^{2}$ \\ ${ }^{1}$ Czech Academy of Sciences, Institute of Mathematics, Žitná 25, 11567 Praha 1, Czech Republic \\ ${ }^{2}$ Université du Sud-Toulon-Var, BP 20132, 83957 La Garde, France \\ Correspondence should be addressed to Jiří Neustupa; neustupa@math.cas.cz
}

Received 15 November 2017; Accepted 22 January 2018; Published 1 March 2018

Academic Editor: Giovanna Bonfanti

Copyright ( 2018 Jiří Neustupa and Patrick Penel. This is an open access article distributed under the Creative Commons Attribution License, which permits unrestricted use, distribution, and reproduction in any medium, provided the original work is properly cited.

\begin{abstract}
The paper shows that the regularity up to the boundary of a weak solution $\mathbf{v}$ of the Navier-Stokes equation with generalized Navier's slip boundary conditions follows from certain rate of integrability of at least one of the functions $\zeta_{1},\left(\zeta_{2}\right)_{+}$(the positive part of $\zeta_{2}$ ), and $\zeta_{3}$, where $\zeta_{1} \leq \zeta_{2} \leq \zeta_{3}$ are the eigenvalues of the rate of deformation tensor $\mathbb{D}(\mathbf{v})$. A regularity criterion in terms of the principal invariants of tensor $\mathbb{D}(\mathbf{v})$ is also formulated.
\end{abstract}

\section{Introduction}

1.1. Navier-Stokes' Initial-Boundary Value Problem. We assume that $\Omega$ is a bounded domain in $\mathbb{R}^{3}$ with a smooth boundary and $T$ is a given positive number. The motion of a viscous incompressible fluid with constant density (which is for simplicity assumed to be equal to one) in domain $\Omega$ in the time interval $(0, T)$ is described by the Navier-Stokes equations:

$$
\begin{aligned}
\partial_{t} \mathbf{v}+\mathbf{v} \cdot \nabla \mathbf{v} & =-\nabla p+\operatorname{div}[2 \nu \mathbb{D}(\mathbf{v})]+\mathbf{f}, \\
\operatorname{div} \mathbf{v} & =0
\end{aligned}
$$

(in $\Omega \times(0, T)$ ) for the unknowns $\mathbf{v} \equiv\left(v_{1}, v_{2}, v_{3}\right)$ and $p$ (the velocity and the pressure). Symbol $v$ denotes the kinematic coefficient of viscosity (it is supposed to be a positive constant) and $\mathbb{D}(\mathbf{v}):=(\nabla \mathbf{v})_{\text {sym }}:=(1 / 2)\left[\nabla \mathbf{v}+(\nabla \mathbf{v})^{T}\right]$ is the socalled rate of deformation tensor. In this paper, we consider (1) and (2) with generalized Navier's slip boundary conditions:

$$
\begin{array}{r}
\mathbf{v} \cdot \mathbf{n}=0, \\
{[2 \nu \mathbb{D}(\mathbf{v}) \cdot \mathbf{n}]_{\tau}+\mathbb{K} \cdot \mathbf{v}=\mathbf{0}}
\end{array}
$$

(on $\partial \Omega \times(0, T)$ ). Here, $\mathbf{n}$ is the outer normal vector on $\partial \Omega$, subscript $\tau$ denotes the tangential component, and $\mathbb{K}$ is a nonnegative 2 nd-order tensor defined a.e. on $\partial \Omega$ such that $\mathbb{K}(\mathbf{x}) \cdot \mathbf{a}$ is tangential to $\partial \Omega$ at point $\mathbf{x} \in \partial \Omega$ if vector $\mathbf{a}$ is tangential to $\partial \Omega$ at point $\mathbf{x}$. Condition (4) generalizes the "classical" Navier boundary condition $[2 \nu \mathbb{D}(\mathbf{v}) \cdot \mathbf{n}]_{\tau}+\kappa \mathbf{v}=\mathbf{0}$, where $\kappa \geq 0$ is the coefficient of friction between the fluid and the boundary. The replacement of $\kappa \mathbf{v}$ by $\mathbb{K} \cdot \mathbf{v}$ reflects the fact that the microscopic structure of $\partial \Omega$ can vary from point to point, it need not produce the same resistance in all tangential directions, and it may therefore divert the flow to the side. In this paper, we assume that $\mathbb{K}$ in (4) is a trace (on $\partial \Omega$ ) of a tensor-valued function from $W^{1,2}(\Omega)^{3 \times 3}$, which is also denoted by $\mathbb{K}$. Problem (1)-(4) is completed by the initial condition

$$
\left.\mathbf{v}\right|_{t=0}=\mathbf{v}_{0} \quad \text { in } \Omega \text {. }
$$

1.2. Shortly on Regularity Criteria for Weak Solutions to System (1) and (2). Existence of a global regular solution and uniqueness of a weak solution are still the fundamental open questions in the theory of the Navier-Stokes equation in 3D. There exist a series of a posteriori assumptions on weak solutions that exclude the development of possible 
singularities. (They are usually called the "criteria of regularity.") The assumptions concern various quantities, like the velocity or some of its components (see, e.g., [1-4]), the gradient of velocity or some of its components (see, e.g., $[3,5]$ ), the vorticity or only two of its components (see, e.g., $[1,6]$ ), the direction of vorticity (see $[7,8]$ ), and the pressure (see, e.g., [9-11]). The absence of a blow-up (i.e., the nonexistence of singularities) in a weak solution has also been proven under certain assumptions on the integrability of the positive part of the middle eigenvalue of the rate of deformation tensor $\mathbb{D}(\mathbf{v})$ in [12].

Most of the known regularity criteria can be applied in the case when either $\Omega=\mathbb{R}^{3}$ (like those from $[1,3,5]$ ) or they exclude singularities in the interior of $\Omega$, but not the singularities on the boundary. (This concerns, e.g., the criteria from $[2,12]$.) As to criteria, valid up to the boundary, we can cite, for example, the papers [13] (where the socalled suitable weak solution is shown to be bounded locally near the boundary if it satisfies Serrin's conditions near the boundary and the trace of the pressure is bounded on the boundary), [14] (where an analogy of the well-known Caffarelli-Kohn-Nirenberg criterion for the regularity of a suitable weak solution at the point $\left(\mathbf{x}_{0}, t_{0}\right) \in \Omega \times(0, T)$, e.g., [15], is also proven for points on a flat part of the boundary), and $[16,17]$ (for some generalizations of the criterion from [14], however, also valid only on a flat part of the boundary). A generalization of the criterion from [14] for points $\left(\mathbf{x}_{0}, t_{0}\right)$ on a "smooth" curved part of the boundary can be found in paper [18]. In paper [19], the author shows that if a weak solution satisfies Serrin's integrability conditions in a neighborhood of a "smooth" part of the boundary then the solution is regular up to this part of the boundary. In all these papers, the authors used the no-slip boundary condition $\mathbf{v}=\mathbf{0}$ on $\partial \Omega \times(0, T)$ (or on the relevant part of this set).

1.3. On the Results of This Paper. In Section 2 of this paper, we consider (1) and (2) with generalized Navier's boundary conditions (3) and (4) and we prove results analogous to those from [12], however, extended so that they hold up to the boundary of $\Omega$. (See Theorem 1.)

Note that while the regularity criteria that consider some components of the velocity or the velocity gradient depend on the observer's frame, the criterion that uses the eigenvalues of tensor $\mathbb{D}(\mathbf{v})$ is frame indifferent. Also note that the study of regularity of a weak solution in the neighborhood of the boundary requires a special technique, which is subtler than the one applied in the interior and closely connected with the used boundary conditions. This can be, for example, documented by the fact that the same result as the one obtained in Section 2 and stated in Theorem 1, for system (1) and (2) with the no-slip boundary condition, is not known.

1.4. Notation. Vector functions and spaces of vector functions are denoted by boldface letters.

(i) The norms of scalar- or vector- or tensor-valued functions with components in $L^{q}(\Omega)\left(\operatorname{resp} ., W^{k, l}(\Omega)\right)$ are denoted by $\|\cdot\|_{q}\left(\right.$ resp., $\left.\|\cdot\|_{k, l}\right)$. The norm in $\mathbf{L}^{2}(\partial \Omega)$ is denoted by $\|\cdot\|_{2 ; \partial \Omega}$. Norms in other spaces on $\partial \Omega$ are denoted by analogy. (ii) $\mathbf{L}_{\sigma}^{2}(\Omega)$ is the closure in $\mathbf{L}^{2}(\Omega)$ of the linear space of all infinitely differentiable divergence-free vector functions with a compact support in $\Omega$. The orthogonal projection of $\mathbf{L}^{2}(\Omega)$ onto $\mathbf{L}_{\sigma}^{2}(\Omega)$ is denoted by $P_{\sigma}$.

(iii) $\mathbf{W}_{\sigma}^{1,2}(\Omega):=\mathbf{W}^{1,2}(\Omega) \cap \mathbf{L}_{\sigma}^{2}(\Omega)$. We denote by $\mathbf{W}_{\sigma}^{-1,2}(\Omega)$ the dual space to $\mathbf{W}_{\sigma}^{1,2}(\Omega)$ and by $\langle\cdot, \cdot\rangle_{\Omega}$ the duality between elements of $\mathbf{W}_{\sigma}^{-1,2}(\Omega)$ and $\mathbf{W}_{\sigma}^{1,2}(\Omega)$.

(iv) \|\|$\cdot \|_{r, s ;\left(t^{\prime}, t^{\prime \prime}\right)}$ denotes the norm of a vector-valued or tensor-valued function with the components in $L^{r}\left(t^{\prime}, t^{\prime \prime} ; L^{s}(\Omega)\right)$.

1.5. A Weak Solution of Problem (1)-(5) and Theorem on Structure. For $\mathbf{v}_{0} \in \mathbf{L}_{\sigma}^{2}(\Omega)$ and $\mathbf{f} \in L^{2}\left(0, T ; \mathbf{W}_{\sigma}^{-1,2}(\Omega)\right)$, a function $\mathbf{v} \in L^{2}\left(0, T ; \mathbf{W}_{\sigma}^{1,2}(\Omega)\right) \cap L^{\infty}\left(0, T ; \mathbf{L}_{\sigma}^{2}(\Omega)\right)$ is called a weak solution of problem (1)-(5) if it satisfies

$$
\begin{aligned}
\int_{0}^{T} & \int_{\Omega}\left\{-\partial_{t} \phi \cdot \mathbf{v}+\mathbf{v} \cdot \nabla \mathbf{v} \cdot \boldsymbol{\phi}+2 v \mathbb{D}(\mathbf{v}): \nabla \phi\right\} \mathrm{d} \mathbf{x} \mathrm{d} t \\
& +\int_{0}^{T} \int_{\partial \Omega}(\mathbb{K} \cdot \mathbf{v}) \cdot \phi \mathrm{d} S \mathrm{~d} t \\
= & \int_{0}^{T}\langle\mathbf{f}, \phi\rangle_{\Omega} \mathrm{d} t+\int_{\Omega} \mathbf{v}_{0} \cdot \phi(\cdot, 0) \mathrm{d} \mathbf{x}
\end{aligned}
$$

for all infinitely differentiable divergence-free vector functions $\phi$ in $\bar{\Omega} \times[0, T]$, such that $\phi \cdot \mathbf{n}=0$ on $\partial \Omega \times$ $[0, T]$ and $\phi(\cdot, T)=\mathbf{0}$. The existence of a weak solution of problem (1)-(3) and (5) with "classical" Navier's boundary condition $[2 \gamma \mathbb{D}(\mathbf{v}) \cdot \mathbf{n}]_{\tau}+\kappa \mathbf{v}=\mathbf{0}$ follows, for example, from papers $[20,21]$. (Note that the more general case of a time-varying domain $\Omega$ is considered in [21].) Applying the same methods, one can also extend the existential results from $[20,21]$ to problem (1)-(5), which includes generalized Navier boundary condition (4). Moreover, by analogy with the Navier-Stokes equations with the no-slip boundary condition $\mathbf{v}=\mathbf{0}$ on $\partial \Omega \times(0, T)$, the weak solution can be constructed so that it satisfies the so-called strong energy inequality:

$$
\begin{array}{r}
\|\mathbf{v}(t)\|_{2}^{2}+4 v \int_{s}^{t} \int_{\Omega}|\mathbb{D}(\mathbf{v}(\vartheta))|^{2} \mathrm{~d} \mathbf{x} \mathrm{d} \vartheta \\
+2 \int_{s}^{t} \int_{\partial \Omega} \mathbf{v}(\vartheta) \cdot \mathbb{K} \cdot \mathbf{v}(\vartheta) \mathrm{d} S \mathrm{~d} \vartheta \\
\leq\|\mathbf{v}(s)\|_{2}^{2}+\int_{s}^{t}\langle\mathbf{f}(\vartheta), \mathbf{v}(\vartheta)\rangle_{\Omega} \mathrm{d} \vartheta
\end{array}
$$

for a.a $s \in(0, T)$ and all $t \in(s, T)$.

In contrast to Navier-Stokes equations (1) and (2) with the no-slip boundary condition, whose theory is relatively well elaborated, the equations with generalized Navier's boundary conditions (3) and (4) have not yet been given so much attention. This is why a series of important results, well known from the theory of equations (1), (2) with the noslip boundary condition, have not been explicitly proven in literature for equations with boundary conditions (3), (4), although many of them can be obtained in a similar or 
almost the same way. This concerns except others the local in time existence of a strong solution (here, however, one can cite the papers $[20,22]$, where the local in time existence of a strong solution is proven in the case when $\mathbb{K}=\kappa \rrbracket$, $\kappa \geq 0$ ), the uniqueness of the weak solution, and the socalled theorem on structure. This theorem states that if the specific volume force $\mathbf{f}$ is at least in $L^{2}\left(0, T ; \mathbf{L}^{2}(\Omega)\right)$ and $\mathbf{v}$ is a weak solution of the Navier-Stokes problem with the no-slip boundary condition, satisfying the strong energy inequality, then $(0, T)=\bigcup_{\gamma \in \Gamma}\left(a_{\gamma}, b_{\gamma}\right) \cup G$, where set $\Gamma$ is at most countable, the intervals $\left(a_{\gamma}, b_{\gamma}\right)$ are pairwise disjoint, the 1D Lebesgue measure of set $G$ is zero, and solution $\mathbf{v}$ coincides with a strong solution in the interior of each of the time intervals $\left(a_{\gamma}, b_{\gamma}\right)$. (See, e.g., [23] for more details.) In this paper, we also use the theorem on structure, but we apply it to the Navier-Stokes problem with boundary conditions (3), (4). (As is mentioned above, the validity of the theorem for the problem with boundary conditions (3), (4) can be proven by means of similar arguments as in the case of the no-slip boundary condition.)

\section{Regularity up to the Boundary in Dependence on Eigenvalues or Principal Invariants of Tensor $\mathbb{D}(\mathbf{v})$}

The main theorem of this section is as follows.

Theorem 1. Let $\mathbf{f} \in L^{2}\left(0, T ; \mathbf{L}^{2}(\Omega)\right)$ and $\mathbb{K} \in W^{1,2}(\Omega)^{3 \times 3}$ be a 2nd-order tensor-valued function such that, for a.a. $\mathbf{x} \in$ $\partial \Omega, \mathbb{K}(\mathbf{x})$ is nonnegative and $\mathbb{K}(\mathbf{x}) \cdot \mathbf{a}$ is tangential to $\partial \Omega$ at point $\mathbf{x}$ if vector $\mathbf{a}$ is tangential to $\partial \Omega$ at point $\mathbf{x}$. Let $\mathbf{v}$ be a weak solution of problem (1)-(5), satisfying the strong energy inequality. Suppose that $\zeta_{1} \leq \zeta_{2} \leq \zeta_{3}$ are the eigenvalues of tensor $\mathbb{D}(\mathbf{v})$ and

(i) one of the functions $\zeta_{1},\left(\zeta_{2}\right)_{+}, \zeta_{3}$ belongs to $L^{r}(0, T$; $\left.\mathbf{L}^{s}(\Omega)\right)$ for some $r \in[1, \infty], s \in(3 / 2, \infty]$, satisfying $2 / r+3 / s=2$.

Then the norm $\|\nabla \mathbf{v}(t)\|_{2}$ is bounded for $t \in(\epsilon, T)$ for any $\epsilon>0$. Moreover, if $\mathbf{v}_{0} \in \mathbf{W}_{\sigma}^{1,2}(\Omega)$ then $\|\nabla \mathbf{v}(\cdot, t)\|_{2}$ is bounded on the whole interval $(0, T)$.

The conclusion of the theorem implies that the solution $\mathbf{v}$ has no singular points in $\Omega \times(0, T)$.

Remark 2. The eigenvalues $\zeta_{1}, \zeta_{2}, \zeta_{3}$ are all real and they are functions of $\mathbf{x}$ and $t$, because the tensor $\mathbb{D}(\mathbf{v})$ is symmetric and depends on $\mathbf{x}$ and $t$. Since the dynamic stress tensor $\mathbb{T}_{\mathrm{d}}(\mathbf{v})$ equals $2 v \mathbb{D}(\mathbf{v})$ in the Newtonian fluid, the eigenvalues of $\mathbb{D}(\mathbf{v})$ coincide, up to the factor $2 v$, with the principal dynamic stresses. The eigenvalues are the roots of the characteristic equation of tensor $\mathbb{D}(\mathbf{v})$, that is, the equation $F(\zeta):=\zeta^{3}-$ $E_{1} \zeta^{2}+E_{2} \zeta-E_{3}=0$, where $E_{1}, E_{2}, E_{3}$ are the principal invariants of $\mathbb{D}(\mathbf{v})$. The invariant $E_{1}$ is equal to zero, because $\operatorname{Tr} \mathbb{D}(\mathbf{v}) \equiv \zeta_{1}+\zeta_{2}+\zeta_{3}=0$. Furthermore,

$$
E_{2}=\zeta_{1} \zeta_{2}+\zeta_{2} \zeta_{3}+\zeta_{3} \zeta_{1}=-\frac{1}{2}\left(\zeta_{1}^{2}+\zeta_{2}^{2}+\zeta_{3}^{2}\right) \leq 0
$$

and $E_{3}=\zeta_{1} \zeta_{2} \zeta_{3}$. Put $\zeta^{*}:=\sqrt{-(1 / 3) E_{2}} \cdot\left( \pm \zeta^{*}\right.$ are the points on the $\zeta$-axis, where $F^{\prime}(\zeta)=0$.) Obviously, $E_{2}=0$ implies $\zeta_{1}=\zeta_{2}=\zeta_{3}=0$. Thus, assume that $E_{2}<0$. Then sgn $\zeta_{2}=$ $\operatorname{sgn}\left(-E_{3}\right)$. The root $\zeta_{2}$ lies between $\zeta^{* *}:=E_{3} / E_{2}$ (the point where the tangent line to the graph of $F$ at the point $\left(0,-E_{3}\right)$ intersects the $\zeta$-axis) and $(3 / 2) E_{3} / E_{2} \equiv(3 / 2) \zeta^{* *}$ (the point where the line connecting the points $\left(0,-E_{3}\right)$ and $\left(\left(\zeta^{*}, F\left(\zeta^{*}\right)\right)\right.$ (if $\left.E_{3}<0\right)$ or $\left(-\zeta^{*}, F\left(-\zeta^{*}\right)\right)$ (if $\left.E_{3}>0\right)$ intersects the $\zeta$-axis). The positive part of $\zeta_{2}$ satisfies $0 \leq\left(\zeta_{2}\right)_{+} \leq(3 / 2) \zeta_{+}^{* *}$. Define function $\mathscr{E}$ by the formula

$$
\mathscr{E}(\mathbf{x}, t):= \begin{cases}0 & \text { if } E_{2}(\mathbf{x}, t)=0, \\ \zeta_{+}^{* *}(\mathbf{x}, t) \equiv\left[\frac{E_{3}(\mathbf{x}, t)}{E_{2}(\mathbf{x}, t)}\right]_{+} & \text {if } E_{2}(\mathbf{x}, t)<0 .\end{cases}
$$

Now, we observe that the statement of Theorem 1 is also valid if condition (i) is replaced by the condition

(ii) $\mathscr{E} \in L^{r}\left(0, T ; \mathbf{L}^{s}(\Omega)\right)$ for some $r \in[1, \infty], s \in(3 / 2, \infty]$, satisfying $2 / r+3 / s=2$.

Proof of Theorem 1. We assume that $t_{0}$ is in one of the intervals $\left(a_{\gamma}, b_{\gamma}\right)$ (see Section 1.5) and $t_{0}<t<b_{\gamma}$. We may assume without the loss of generality that $b_{\gamma}$ is the largest number $\leq$ $T$ such that $\mathbf{v}$ is "smooth" on the time interval $\left(t_{0}, b_{\gamma}\right)$. Then there are two possibilities: (a) the first singularity of solution $\mathbf{v}$ (after the time instant $t_{0}$ ) develops at the time $b_{\gamma}$ or (b) no singularity of $\mathbf{v}$ develops at any time $t \in\left(t_{0}, T\right]$. Assume, by contradiction, that the possibility (a) takes place. In this case, $b_{\gamma}$ is called the epoch of irregularity.

There exists an associated pressure $p$ so that $\mathbf{v}$ and $p$ satisfy (1), (2) a.e. in $\Omega \times\left(a_{\gamma}, b_{\gamma}\right)$. Multiplying (1) by $P_{\sigma} \Delta \mathbf{v}$ and integrating in $\Omega$, we obtain

$$
\int_{\Omega} \partial_{t} \mathbf{v} \cdot P_{\sigma} \Delta \mathbf{v} \mathrm{d} \mathbf{x}+\int_{\Omega} \mathbf{v} \cdot \nabla \mathbf{v} \cdot P_{\sigma} \Delta \mathbf{v} \mathrm{d} \mathbf{x}=\nu\left\|P_{\sigma} \Delta \mathbf{v}\right\|_{2}^{2} .
$$

The first integral on the left hand side can be treated as follows:

$$
\begin{aligned}
\int_{\Omega} \partial_{t} \mathbf{v} \cdot P_{\sigma} \Delta \mathbf{v} \mathrm{d} \mathbf{x}= & \int_{\Omega} \partial_{t} \mathbf{v} \cdot \Delta \mathbf{v} \mathrm{d} \mathbf{x} \\
= & 2 \int_{\Omega} \partial_{t} \mathbf{v} \cdot \operatorname{div} \mathbb{D}(\mathbf{v}) \mathrm{d} \mathbf{x} \\
= & 2 \int_{\partial \Omega} \partial_{t} \mathbf{v} \cdot[\mathbb{D}(\mathbf{v}) \cdot \mathbf{n}] \mathrm{d} S \\
& -2 \int_{\Omega} \partial_{t} \nabla \mathbf{v}: \mathbb{D}(\mathbf{v}) \mathrm{d} \mathbf{x} \\
= & -\frac{1}{v} \int_{\partial \Omega} \partial_{t} \mathbf{v} \cdot(\mathbb{K} \cdot \mathbf{v}) \mathrm{d} S \\
& -\frac{\mathrm{d}}{\mathrm{d} t} \int_{\Omega}|\mathbb{D}(\mathbf{v})|^{2} \mathrm{~d} \mathbf{x} \\
= & -\frac{1}{2 v} \frac{\mathrm{d}}{\mathrm{d} t} \int_{\partial \Omega} \mathbf{v} \cdot \mathbb{K} \cdot \mathbf{v} \mathrm{d} S \\
& -\frac{\mathrm{d}}{\mathrm{d} t}\|\mathbb{D}(\mathbf{v})\|_{2}^{2} \cdot
\end{aligned}
$$

Before we estimate the second integral on the left hand side of (10), we recall some inequalities: 
$(\alpha)$ the Friedrichs-type inequality $\|\mathbf{u}\|_{2} \leq c_{1}\|\nabla \mathbf{u}\|_{2}$ (see, e.g., [24, Exercise II.5.15]), satisfied for all functions $\mathbf{u} \in \mathbf{W}^{1,2}(\Omega)$ such that $\mathbf{u} \cdot \mathbf{n}=0$ on $\partial \Omega$

( $\beta$ ) The inequality $\left\|\nabla^{2} \mathbf{u}\right\|_{2} \leq c_{2}\left(\|\Delta \mathbf{u}\|_{2}+\|\mathbf{u}\|_{2}\right)$, which holds for $\mathbf{u} \in \mathbf{W}^{2,2}(\Omega)$ that satisfy Navier's boundary conditions (3), (4) (following from [20, Theorem 3.1]).

The Helmholtz decomposition of $\Delta \mathbf{u}$ is $\Delta \mathbf{u}=P_{\sigma} \Delta \mathbf{u}+\nabla \varphi$, where

$$
\begin{aligned}
& \text { (a) } \Delta \varphi=0 \quad \text { in } \Omega, \\
& \text { (b) } \frac{\partial \varphi}{\partial \mathbf{n}}=\Delta \mathbf{u} \cdot \mathbf{n} \quad \text { on } \partial \Omega .
\end{aligned}
$$

The next lemma brings the crucial estimates of $\|\nabla \varphi\|_{2}$ and $\|\mathbf{v}\|_{2,2}$.

Lemma 3. There exist $c_{3}, c_{4}, c_{5} c_{6}>0$ such that if $\mathbf{u}$ is a divergence-free function from $\mathbf{W}^{2,2}(\Omega)$ that satisfies boundary conditions (3), (4) and $\varphi$ is a solution of the Neumann problem (12) then

$$
\begin{gathered}
\|\nabla \varphi\|_{2} \leq c_{3}\|\nabla(\mathbb{K} \cdot \mathbf{u})\|_{2}+c_{4}\|\mathbf{u}\|_{1,2}, \\
\|\mathbf{u}\|_{2,2} \leq c_{5}\left\|P_{\sigma} \Delta \mathbf{u}\right\|_{2}+c_{6}\|\mathbf{u}\|_{2} .
\end{gathered}
$$

Proof. The right hand side $\Delta \mathbf{u} \cdot \mathbf{n}$ in the boundary condition $((12)(b))$ equals

$$
\begin{gathered}
-\operatorname{curl}^{2} \mathbf{u} \cdot \mathbf{n}=-\operatorname{curl}\left[(\operatorname{curl} \mathbf{u})_{\tau}\right] \cdot \mathbf{n}-\operatorname{curl}\left[(\operatorname{curl} \mathbf{u})_{n}\right] \\
\cdot \mathbf{n}=-\operatorname{curl}\left[(\operatorname{curl} \mathbf{u})_{\tau}\right] \cdot \mathbf{n} .
\end{gathered}
$$

(The vector field $\operatorname{curl}\left[(\operatorname{curl} \mathbf{u})_{n}\right]$ is tangential because $(\operatorname{curl} \mathbf{u})_{n}$ is normal. Hence the term $\operatorname{curl}\left[(\operatorname{curl} \mathbf{u})_{n}\right] \cdot \mathbf{n}$ equals zero on $\partial \Omega$.) The tangential component of curl $\mathbf{u}$, that is, $(\operatorname{curl} \mathbf{u})_{\tau}$, equals $\mathbf{n} \times$ curl $\mathbf{u} \times \mathbf{n}$. In order to express curl $\mathbf{u} \times \mathbf{n}$, we apply the formula $[2 \mathbb{D}(\mathbf{u}) \cdot \mathbf{n}]_{\tau}=\operatorname{curl} \mathbf{u} \times \mathbf{n}-2 \mathbf{u} \cdot \nabla \mathbf{n}$ (see, e.g., [20]). Hence, using also the boundary condition (4), we obtain

$$
\begin{aligned}
(\operatorname{curl} \mathbf{u})_{\tau} & =\mathbf{n} \times(\operatorname{curl} \mathbf{u} \times \mathbf{n}) \\
& =\mathbf{n} \times\left([2 \mathbb{D}(\mathbf{u}) \cdot \mathbf{n}]_{\tau}+2 \mathbf{u} \cdot \nabla \mathbf{n}\right) \\
& =\mathbf{n} \times\left(-\frac{1}{\nu} \mathbb{K} \cdot \mathbf{u}+2 \mathbf{u} \cdot \nabla \mathbf{n}\right) .
\end{aligned}
$$

Thus, boundary condition $((12)(b))$ takes the form

$$
\frac{\partial \varphi}{\partial \mathbf{n}}=-\operatorname{curl}\left[\mathbf{n} \times\left(-\frac{1}{\nu} \mathbb{K} \cdot \mathbf{u}+2 \mathbf{u} \cdot \nabla \mathbf{n}\right)\right] \cdot \mathbf{n} .
$$

In comparison to ((12)(b)), the right hand side of (17) contains only the first-order derivatives of $\mathbf{u}$. The classical theory of solution of the Neumann problem now implies that

$$
\begin{aligned}
& \|\nabla \varphi\|_{2} \\
& \quad \leq C\left\|\operatorname{curl}\left[\mathbf{n} \times\left(-\frac{1}{\nu} \mathbb{K} \cdot \mathbf{u}+2 \mathbf{u} \cdot \nabla \mathbf{n}\right)\right] \cdot \mathbf{n}\right\|_{-1 / 2,2 ; \partial \Omega} .
\end{aligned}
$$

(We use $C$ as a generic constant.) The right hand side can be estimated by means of continuity of the linear operator, acting from the space $\mathbf{L}_{\text {div }}^{2}(\Omega)$ (which is the space function $\mathbf{w} \in \mathbf{L}^{2}(\Omega)$, whose divergence in the sense of distributions is in $L^{2}(\Omega)$, with the norm $\left.\|\mathbf{w}\|_{2}+\|\operatorname{div} \mathbf{w}\|_{2}\right)$ to $W^{-1 / 2,2}(\partial \Omega)$, which assigns to "smooth" functions $\mathbf{w} \in \mathbf{L}_{\text {div }}^{2}(\Omega)$ the normal component $\mathbf{w} \cdot \mathbf{n}$. Thus, we obtain the estimate

$$
\|\nabla \varphi\|_{2} \leq C\left\|\operatorname{curl}\left[\mathbf{n} \times\left(-\frac{1}{v} \mathbb{K} \cdot \mathbf{u}+2 \mathbf{u} \cdot \nabla \mathbf{n}\right)\right] \cdot \mathbf{n}\right\|_{2},
$$

(where $C=C(\Omega, v)$ ) which yields (13). Furthermore, $\|\Delta \mathbf{u}\|_{2} \leq$ $\left\|P_{\sigma} \Delta \mathbf{u}\right\|_{2}+\|\nabla \varphi\|_{2}$. Estimating the norm $\|\nabla \varphi\|_{2}$ by means of (13), we get

$$
\begin{aligned}
\|\mathbf{u}\|_{2,2} & \leq C\|\Delta \mathbf{u}\|_{2} \\
& \leq C\left(\left\|P_{\sigma} \Delta \mathbf{u}\right\|_{2}+\|\nabla(\mathbb{K} \cdot \mathbf{u})\|_{2}+\|\mathbf{u}\|_{2}\right) .
\end{aligned}
$$

The norm of $\nabla(\mathbb{K} \cdot \mathbf{v})$ satisfies

$$
\begin{aligned}
\|\nabla(\mathbb{K} \cdot \mathbf{v})\|_{2} & \leq\|\nabla \mathbb{K}\|_{2}\|\mathbf{u}\|_{\infty}+\|\mathbb{K}\|_{6}\|\nabla \mathbf{u}\|_{3} \leq C\|\mathbf{u}\|_{1, q} \\
& \leq \epsilon\|\mathbf{u}\|_{2,2}+C(\epsilon)\|\mathbf{u}\|_{2}
\end{aligned}
$$

for any $q \epsilon(3,6)$ and $\epsilon>0$ due to the imbedding $\mathbf{W}^{2,2}(\Omega) \hookrightarrow \hookrightarrow \mathbf{W}^{1, q}(\Omega) \hookrightarrow \mathbf{L}^{\infty}(\Omega)$. Hence

$$
\|\mathbf{u}\|_{2,2} \leq C\left\|P_{\sigma} \Delta \mathbf{u}\right\|_{2}+C \epsilon\|\mathbf{u}\|_{2,2}+C(\epsilon)\|\mathbf{u}\|_{2} .
$$

Choosing $\epsilon$ sufficiently small, we obtain (14).

Continuation of the Proof of Theorem 1. The second integral in (10) satisfies

$$
\begin{aligned}
\int_{\Omega} \mathbf{v} \cdot \nabla \mathbf{v} \cdot P_{\sigma} \Delta \mathbf{v} \mathrm{d} \mathbf{x}= & \int_{\Omega} \mathbf{v} \cdot \nabla \mathbf{v} \cdot \Delta \mathbf{v} \mathrm{d} \mathbf{x} \\
& -\int_{\Omega} \mathbf{v} \cdot \nabla \mathbf{v} \cdot \nabla \varphi \mathrm{d} \mathbf{x} .
\end{aligned}
$$

The second term on the right hand side can be estimated by means of Lemma 3, (21), and (14):

$$
\begin{aligned}
& \left|\int_{\Omega} \mathbf{v} \cdot \nabla \mathbf{v} \cdot \nabla \varphi \mathrm{d} \mathbf{x}\right| \leq\|\mathbf{v}\|_{\infty}\|\nabla \mathbf{v}\|_{2}\|\nabla \varphi\|_{2} \\
& \quad \leq C\|\mathbf{v}\|_{\infty}\|\nabla \mathbf{v}\|_{2}\left(\|\nabla(\mathbb{K} \cdot \mathbf{v})\|_{2}+\|\mathbf{v}\|_{1,2}\right) \\
& \quad \leq C\|\mathbf{v}\|_{2,2}\|\nabla \mathbf{v}\|_{2}\left(\epsilon\|\mathbf{v}\|_{2,2}+C(\epsilon)\|\mathbf{v}\|_{1,2}\right) \\
& \quad \leq \delta\left\|P_{\sigma} \Delta \mathbf{v}\right\|_{2}^{2}+C(\delta)\|\nabla \mathbf{v}\|_{2}^{4},
\end{aligned}
$$

where $\delta>0$ can be chosen arbitrarily small. The first term on the right hand side of (23) equals

$$
\begin{aligned}
& \int_{\partial \Omega}(\mathbf{v} \cdot \nabla \mathbf{v}) \cdot(\mathbf{n} \cdot \nabla \mathbf{v}) \mathrm{d} S-\int_{\Omega} \nabla(\mathbf{v} \cdot \nabla \mathbf{v}): \nabla \mathbf{v} \mathrm{d} S \\
& \quad \equiv I_{1}+I_{2}-I_{3},
\end{aligned}
$$

where $I_{3}$ denotes the last integral on the left hand side and

$$
\begin{aligned}
& I_{1}:=\int_{\partial \Omega}[\mathbf{v} \cdot \nabla \mathbf{v}]_{n} \cdot(\mathbf{n} \cdot \nabla \mathbf{v}) \mathrm{d} S, \\
& I_{2}:=\int_{\partial \Omega}[\mathbf{v} \cdot \nabla \mathbf{v}]_{\tau} \cdot(\mathbf{n} \cdot \nabla \mathbf{v}) \mathrm{d} S .
\end{aligned}
$$

(Subscripts $n$ and $\tau$ denote the normal and tangential components, resp.) Applying the inequalities in $(\alpha)$ and $(\beta)$, 
Lemma 3 and the boundary conditions (3), (4), the integrals $I_{1}, I_{2}$, and $I_{3}$ can be treated as follows:

$$
\begin{aligned}
I_{1}= & \int_{\partial \Omega}[\mathbf{v} \cdot \nabla \mathbf{v}]_{n} \cdot(\mathbf{n} \cdot \nabla \mathbf{v})_{n} \mathrm{~d} \mathbf{x} \\
= & \int_{\partial \Omega}\left[v_{j}\left(\partial_{j} v_{l}\right) n_{l}\right]\left[n_{k}\left(\partial_{k} v_{m}\right) n_{m}\right] \mathrm{d} S \\
= & \int_{\partial \Omega}\left[v_{j} \partial_{j}\left(v_{l} n_{l}\right)-v_{j} v_{l}\left(\partial_{j} n_{l}\right)\right]\left[n_{k}\left(\partial_{k} v_{m}\right) n_{m}\right] \mathrm{d} S \\
= & -\int_{\partial \Omega}\left[v_{j} v_{l}\left(\partial_{j} n_{l}\right)\right]\left[n_{k}\left(\partial_{k} v_{m}\right) n_{m}\right] \mathrm{d} S \\
= & -\int_{\Omega} \partial_{m}\left\{\left[v_{j} v_{l}\left(\partial_{j} n_{l}\right)\right]\left[n_{k}\left(\partial_{k} v_{m}\right)\right]\right\} \mathrm{d} \mathbf{x} \\
\leq & C\|\mathbf{v}\|_{\infty}\|\nabla \mathbf{v}\|_{2}^{2} \leq C\|\mathbf{v}\|_{2,2}\|\nabla \mathbf{v}\|_{2}^{2} \\
\leq & C\left(\left\|P_{\sigma} \Delta \mathbf{v}\right\|_{2}+\|\mathbf{v}\|_{2}\right)\|\nabla \mathbf{v}\|_{2}^{2} \\
\leq & \delta\left\|P_{\sigma} \Delta \mathbf{v}\right\|_{2}^{2}+C(\delta)\|\nabla \mathbf{v}\|_{2}^{4}+C, \\
I_{2}= & \int_{\partial \Omega}(\mathbf{v} \cdot \nabla \mathbf{v})_{\tau} \cdot(\mathbf{n} \cdot \nabla \mathbf{v}) \mathrm{d} S \\
= & \int_{\partial \Omega}(\mathbf{v} \cdot \nabla \mathbf{v})_{\tau} \cdot\left(\mathbf{n} \cdot\left[\nabla \mathbf{v}+(\nabla \mathbf{v})^{T}\right]\right) \mathrm{d} S \\
& -\int_{\partial \Omega}(\mathbf{v} \cdot \nabla \mathbf{v})_{\tau} \cdot\left[\mathbf{n} \cdot(\nabla \mathbf{v})^{T}\right] \mathrm{d} S \\
= & \int_{\partial \Omega}(\mathbf{v} \cdot \nabla \mathbf{v})_{\tau} \cdot[2 \mathbb{D}(\mathbf{v}) \cdot \mathbf{n}]_{\tau} \mathrm{d} S \\
& (\mathbf{v} \cdot \nabla \mathbf{v})_{\tau} \cdot[\nabla(\mathbf{n} \cdot \mathbf{v})-\nabla \mathbf{n} \cdot \mathbf{v}] \mathrm{d} S
\end{aligned}
$$

Since $(\mathbf{v} \cdot \nabla \mathbf{v})_{\tau}$ is tangential and $\mathbf{n} \cdot \mathbf{v}=0$ on $\partial \Omega$, the scalar product $(\mathbf{v} \cdot \nabla \mathbf{v})_{\tau} \cdot \nabla(\mathbf{n} \cdot \mathbf{v})$ is equal to zero. Thus, if we also use boundary condition (4), the inequalities in $(\alpha)$ and $(\beta)$, and Lemma 3, we get

$$
\begin{aligned}
& \left|I_{2}\right|=\mid-\frac{1}{v} \int_{\partial \Omega}(\mathbf{v} \cdot \nabla \mathbf{v})_{\tau} \cdot(\mathbb{K} \cdot \mathbf{v}) \mathrm{d} S \\
& \quad+\left.\int_{\partial \Omega}(\mathbf{v} \cdot \nabla \mathbf{v})_{\tau}(\nabla \mathbf{n} \cdot \mathbf{v}) \mathrm{d} S\left|\leq C \int_{\partial \Omega}\right| \mathbf{v}\right|^{2}|\nabla \mathbf{v}|(|\mathbb{K}| \\
& \quad+1) \mathrm{d} S \leq C\|\mathbf{v}\|_{4 ; \partial \Omega}^{2}\|\nabla \mathbf{v}\|_{4 ; \partial \Omega}\left(\|\mathbb{K}\|_{4 ; \partial \Omega}+1\right) \\
& \quad \leq C\|\mathbf{v}\|_{1,2}^{2}\|\mathbf{v}\|_{2,2}\left(\|\mathbb{K}\|_{1,2}+1\right) \leq C\|\nabla \mathbf{v}\|_{2}^{2}\left(\left\|P_{\sigma} \Delta \mathbf{v}\right\|_{2}\right. \\
& \left.\quad+\|\mathbf{v}\|_{2}\right) \leq \delta\left\|P_{\sigma} \Delta \mathbf{v}\right\|_{2}^{2}+C(\delta)\|\nabla \mathbf{v}\|_{2}^{4}+C \\
& I_{3}=\int_{\Omega}\left[\left(\partial_{k} v_{j}\right)\left(\partial_{j} v_{i}\right)\left(\partial_{k} v_{i}\right)+v_{j}\left(\partial_{j k}^{2} v_{i}\right)\left(\partial_{k} v_{i}\right)\right] \mathrm{d} \mathbf{x} \\
& \quad=\int_{\Omega}\left(\partial_{k} v_{j}\right)\left(\partial_{j} v_{i}\right)\left(\partial_{k} v_{i}\right) \mathrm{d} \mathbf{x} .
\end{aligned}
$$

If we denote (for $i, j=1,2,3) d_{i j}:=(1 / 2)\left[\left(\partial_{i} v_{j}\right)+\left(\partial_{j} v_{i}\right)\right]$ (the entries of tensor $\mathbb{D})$ and $s_{i j}:=(1 / 2)\left[\left(\partial_{i} v_{j}\right)-\left(\partial_{j} v_{i}\right)\right]$ (the entries of the skew-symmetric part of $\nabla \mathbf{v}$ ), we obtain

$$
\begin{aligned}
I_{3} & =\int_{\Omega}\left(d_{k j}+s_{k j}\right)\left(d_{j i}+s_{j i}\right)\left(d_{k i}+s_{k i}\right) \mathrm{d} S \\
& =\int_{\Omega}\left[d_{k j} d_{j i} d_{k i}+d_{k j} s_{j i} s_{k i}+d_{j i} s_{k j} s_{k i}+d_{k i} s_{k j} s_{j i}\right] \mathrm{d} \mathbf{x} .
\end{aligned}
$$

As $s_{j i}=-s_{i j}$, we have $d_{k j} s_{j i} s_{k i}+d_{k i} s_{k j} s_{j i}=d_{k j} s_{j i} s_{k i}+d_{k j} s_{k i} s_{i j}=$ 0 . Hence

$$
\begin{aligned}
I_{3} & =\int_{\Omega}\left[d_{k j} d_{j i} d_{i k}+d_{i j} s_{k i} s_{k j}\right] \mathrm{d} \mathbf{x} \\
& =\int_{\Omega} d_{k j} d_{j i} d_{i k} \mathrm{~d} \mathbf{x}-\frac{1}{4} \int_{\Omega} d_{i j} \omega_{i} \omega_{j} \mathrm{~d} \mathbf{x},
\end{aligned}
$$

where $\omega_{i}$ and $\omega_{j}$ are the components of $\boldsymbol{\omega}:=$ curlv. The estimates (27), (29) and the identity (32) yield

$$
\begin{aligned}
\int_{\Omega} \mathbf{v} \cdot \nabla \mathbf{v} \cdot \Delta \mathbf{v} \mathrm{d} \mathbf{x} \leq & 2 \delta\left\|P_{\sigma} \Delta \mathbf{v}\right\|_{2}^{2}+C(\delta)\|\nabla \mathbf{v}\|_{2}^{4}+C \\
& -\int_{\Omega} d_{k j} d_{j i} d_{i k} \mathrm{~d} \mathbf{x} \\
& +\frac{1}{4} \int_{\Omega} d_{i j} \omega_{i} \omega_{j} \mathrm{~d} \mathbf{x} .
\end{aligned}
$$

The integral on the left hand side of (33) can also be treated in another way:

$$
\begin{aligned}
\int_{\Omega} \mathbf{v} \cdot \nabla \mathbf{v} \cdot \Delta \mathbf{v} \mathrm{d} \mathbf{x}= & -\int_{\Omega} \mathbf{v} \cdot \nabla \mathbf{v} \cdot \operatorname{curl}^{2} \mathbf{v} \mathrm{d} \mathbf{x} \\
= & -\int_{\partial \Omega} \mathbf{v} \cdot \nabla \mathbf{v} \cdot(\mathbf{n} \times \operatorname{curl} \mathbf{v}) \mathrm{d} S \\
& -\int_{\Omega} \operatorname{curl}(\mathbf{v} \cdot \nabla \mathbf{v}) \cdot \operatorname{curl} \mathbf{v} \mathrm{d} \mathbf{x} .
\end{aligned}
$$

The integrals on the right hand side can be estimated or modified as follows:

$$
\begin{aligned}
& \left|\int_{\partial \Omega} \mathbf{v} \cdot \nabla \mathbf{v} \cdot(\mathbf{n} \times \mathbf{c u r l} \mathbf{v}) \mathrm{d} S\right| \\
& \quad=\left|\int_{\partial \Omega}(\mathbf{v} \cdot \nabla \mathbf{v}) \cdot\left([2 \mathbb{D} \cdot \mathbf{n}]_{\tau}+2 \mathbf{v} \cdot \nabla \mathbf{n}\right) \mathrm{d} S\right| \\
& \quad=\left|\frac{1}{\nu} \int_{\partial \Omega}(\mathbf{v} \cdot \nabla \mathbf{v}) \cdot(-\mathbb{K} \cdot \mathbf{v}+2 \mathbf{v} \cdot \nabla \mathbf{n}) \mathrm{d} S\right| \\
& \quad \leq C \int_{\partial \Omega}|\mathbf{v}|^{2}|\nabla \mathbf{v}|(|\mathbb{K}|+1) \mathrm{d} S \\
& \quad \leq C\|\mathbf{v}\|_{4 ; \partial \Omega}^{2}\|\nabla \mathbf{v}\|_{2 ; \partial \Omega}\left(\|\mathbb{K}\|_{4 ; \partial \Omega}+1\right) \\
& \quad \leq \delta\left\|P_{\sigma} \Delta \mathbf{v}\right\|_{2}^{2}+C(\delta)\|\nabla \mathbf{v}\|_{2}^{4}+C
\end{aligned}
$$


(by analogy with (29)),

$$
\begin{aligned}
& \int_{\Omega} \operatorname{curl}(\mathbf{v} \cdot \nabla \mathbf{v}) \cdot \operatorname{curl} \mathbf{v} \mathrm{d} \mathbf{x} \\
& \quad=\int_{\Omega}[\mathbf{v} \cdot \nabla \boldsymbol{\omega}-\boldsymbol{\omega} \cdot \nabla \mathbf{v}] \cdot \boldsymbol{\omega} \mathrm{d} \mathbf{x}=-\int_{\Omega} \boldsymbol{\omega} \cdot \nabla \mathbf{v} \cdot \boldsymbol{\omega} \mathrm{d} \mathbf{x} \\
& =-\int_{\Omega} \boldsymbol{\omega} \cdot \mathbb{D}(\mathbf{v}) \cdot \boldsymbol{\omega} \mathrm{d} \mathbf{x}=-\int_{\Omega} d_{i j} \omega_{i} \omega_{j} \mathrm{~d} \mathbf{x} .
\end{aligned}
$$

Multiplying (34)-(36) by $1 / 4$, we get

$$
\begin{aligned}
\frac{1}{4} \int_{\Omega} \mathbf{v} \cdot \nabla \mathbf{v} \cdot \Delta \mathbf{v} \mathrm{d} \mathbf{x} \leq & \frac{\delta}{4}\left\|P_{\sigma} \Delta \mathbf{v}\right\|_{2}^{2}+C(\delta)\|\nabla \mathbf{v}\|_{2}^{4}+C \\
& -\frac{1}{4} \int_{\Omega} d_{i j} \omega_{i} \omega_{j} \mathrm{~d} \mathbf{x} .
\end{aligned}
$$

Summing (33) and (37), we obtain

$$
\begin{aligned}
\frac{5}{4} \int_{\Omega} \mathbf{v} \cdot \nabla \mathbf{v} \cdot \Delta \mathbf{v} \mathrm{d} \mathbf{x} \leq & \frac{9 \delta}{4}\left\|P_{\sigma} \Delta \mathbf{v}\right\|_{2}^{2}+C(\delta)\|\nabla \mathbf{v}\|_{2}^{4}+C \\
& -\int_{\Omega} d_{k j} d_{j i} d_{i k} \mathrm{~d} \mathbf{x} .
\end{aligned}
$$

Dividing this inequality by $5 / 4$, choosing $\delta=(5 / 18) v$, substituting to (10), and expressing the first integral in (10) by means of (11), we obtain

$$
\begin{gathered}
\frac{\mathrm{d}}{\mathrm{d} t}\|\mathbb{D}(\mathbf{v})\|_{2}^{2}+\frac{1}{2 \nu} \frac{\mathrm{d}}{\mathrm{d} t} \int_{\partial \Omega} \mathbf{v} \cdot \mathbb{K} \cdot \mathbf{v} \mathrm{d} S+\frac{v}{2}\left\|P_{\sigma} \Delta\right\|_{2}^{2} \\
\leq-\frac{4}{5} \int_{\Omega} d_{k j} d_{j i} d_{i k} \mathrm{~d} \mathbf{x}+c_{7}\|\nabla \mathbf{v}\|_{2}^{2}\|\mathbb{D}(\mathbf{v})\|_{2}^{2}+c_{8} .
\end{gathered}
$$

The product $d_{j k} d_{k i} d_{i j}$ equals the trace of the tensor $\mathbb{D}(\mathbf{v})^{3}$. It is invariant with respect to rotation of the coordinate system. Hence it can be represented in the system in which $\mathbb{D}(\mathbf{v})$ has the diagonal representation $\mathbb{D}=\left(d_{i j}\right)$ with $d_{i j}=0$ for $i \neq$ $j$ and $d_{11}=\zeta_{1}, d_{22}=\zeta_{2}, d_{33}=\zeta_{3}$, where $\zeta_{1}, \zeta_{3} \zeta_{3}$ are the eigenvalues of tensor $\mathbb{D}(\mathbf{v})$. The eigenvalues are real because $\mathbb{D}(\mathbf{v})$ is symmetric and their sum is zero because of the trace if $\mathbb{D}(\mathbf{v})$ is equal to zero. Then $\operatorname{Tr} \mathbb{D}(\mathbf{v})^{3}=d_{j k} d_{k i} d_{i j}=\zeta_{1}^{3}+\zeta_{2}^{3}+\zeta_{3}^{3}=$ $3 \zeta_{1} \zeta_{2} \zeta_{3}$. We may assume that the eigenvalues are ordered so that $\zeta_{1} \leq \zeta_{2} \zeta_{3}$, which implies that $\zeta_{1} \leq 0$ and $\zeta_{3} \geq 0$. Then inequality (39) takes the form

$$
\begin{aligned}
\frac{\mathrm{d}}{\mathrm{d} t}\|\mathbb{D}(\mathbf{v})\|_{2}^{2}+\frac{1}{2 v} \frac{\mathrm{d}}{\mathrm{d} t} \int_{\partial \Omega} \mathbf{v} \cdot \mathbb{K} \cdot \mathbf{v} \mathrm{d} S+\frac{v}{2}\left\|P_{\sigma} \Delta\right\|_{2}^{2} \\
\leq-\frac{12}{5} \int_{\Omega}\left(-\zeta_{1}\right)\left(\zeta_{2}\right)_{+} \zeta_{3} \mathrm{~d} \mathbf{x}+c_{7}\|\nabla \mathbf{v}\|_{2}^{2}\|\mathbb{D}(\mathbf{v})\|_{2}^{2} \\
\quad+c_{8} .
\end{aligned}
$$

Integrating this inequality on the time interval $\left(t_{0}, t_{1}\right)$, where $t_{0}<t_{1} \leq b_{\gamma}$, we deduce that

$$
\begin{aligned}
& \|\mathbb{D}(\mathbf{v})\|_{\infty, 2 ;\left(t_{0}, t_{1}\right)}^{2}+\frac{\nu}{2}\left\|P_{\sigma} \Delta \mathbf{v}\right\|_{2,2 ;\left(t_{0}, t_{1}\right)}^{2} \\
& \leq c_{9}\left\|\mathbb{D}\left(\mathbf{v}\left(t_{0}\right)\right)\right\|_{2}^{2}+c_{10} \int_{t_{0}}^{t_{1}} \int_{\Omega}\left(-\zeta_{1}\right)\left(\zeta_{2}\right)_{+} \zeta_{3} \mathrm{~d} \mathbf{x} \mathrm{d} \vartheta \\
& \quad+c_{11},
\end{aligned}
$$

where constants $c_{9}, c_{10}, c_{11}$ depend on $\nu, \Omega, c_{7}, c_{8}$, and also the norm $\|\nabla \mathbf{v}\|_{2,2 ;(0, T)}$. Let us further estimate the integral of $\left(-\zeta_{1}\right)\left(\zeta_{2}\right)_{+} \zeta_{3}$ on the right hand side of (39). Assume, for example, that $\left(\zeta_{2}\right)_{+} \in L^{r}\left(0, T ; L^{s}(\Omega)\right)$, where $2 / r+3 / s \leq 1$. Since $\left|\zeta_{i}\right| \leq C|\nabla \mathbf{v}|(i=1,2,3)$, we have

$$
\begin{aligned}
& \int_{t_{0}}^{t_{1}} \int_{\Omega}\left(-\zeta_{1}\right)\left(\zeta_{2}\right)_{+} \zeta_{3} \mathrm{~d} \mathbf{x} \mathrm{d} t \\
& \quad \leq\left\|\left(\zeta_{2}\right)_{+}\right\|_{r, s ;\left(t_{0}, t_{1}\right)}\left\|\zeta_{1} \zeta_{3}\right\|_{r /(r-1), s /(s-1) ;\left(t_{0}, t_{1}\right)} \\
& \quad \leq c_{12}\left\|\left(\zeta_{2}\right)_{+}\right\|_{r, s ;\left(t_{0}, t_{1}\right)}\|\nabla \mathbf{v}\|_{2 r /(r-1), 2 s /(s-1) ;\left(t_{0}, t_{1}\right)}^{2} .
\end{aligned}
$$

Estimating the norm of $\nabla \mathbf{v}$ by means of the inequality

$$
\begin{aligned}
& \|\boldsymbol{g}\|_{\alpha, \beta ;\left(t_{0}, t_{1}\right)} \leq\|g\|_{2,2 ;\left(t_{0}, t_{1}\right)}^{2 / \alpha+3 / \beta-3 / 2}\left(\|g\|_{\infty, 2 ;\left(t_{0}, t_{1}\right)}\right. \\
& \left.\quad+\|g\|_{2,6 ;\left(t_{0}, t_{1}\right)}\right)^{5 / 2-(2 / \alpha+3 / \beta)}
\end{aligned}
$$

which can be proven by means of Hölder's inequality and which is valid for $2 \leq \alpha \leq \infty, 2 \leq \beta \leq 6$, and $3 / 2 \leq$ $2 / \alpha+3 / \beta \leq 5 / 2$, with $\alpha=2 r /(r-1)$ and $\beta=2 s /(s-1)$, we obtain

$$
\begin{aligned}
& \int_{t_{0}}^{t_{1}} \int_{\Omega}\left(-\zeta_{1}\right)\left(\zeta_{2}\right)_{+} \zeta_{3} \mathrm{~d} \mathbf{x} \mathrm{d} t \\
& \quad \leq\left\|\left(\zeta_{2}\right)_{+}\right\|_{\left.r, s ; t_{0}, t_{1}\right)}\left(\|\nabla \mathbf{v}\|_{\infty, 2 ;\left(t_{0}, t_{1}\right)}\right. \\
& \left.\quad+\|\nabla \mathbf{v}\|_{2,6 ;\left(t_{0}, t_{1}\right)}\right)^{2 / r+3 / s} \\
& \quad \leq c_{13}\left\|\left(\zeta_{2}\right)_{+}\right\|_{r, s ;\left(t_{0}, t_{1}\right)}\left(\|\mathbb{D}(\mathbf{v})\|_{\infty, 2 ;\left(t_{0}, t_{1}\right)}^{2}\right. \\
& \left.\quad+\frac{v}{2}\left\|P_{\sigma} \Delta \mathbf{v}\right\|_{2,2 ;\left(t_{0}, t_{1}\right)}^{2}+c_{14}\right) .
\end{aligned}
$$

(The norm $\|\nabla \mathbf{v}\|_{2}$ inside $\|\nabla \mathbf{v}\|_{\infty, 2 ;\left(t_{0}, t_{1}\right)}$ has been estimated by Korn's inequality and the norm $\|\nabla \mathbf{v}\|_{6}$ inside $\|\nabla \mathbf{v}\|_{2,6 ;\left(t_{0}, t_{1}\right)}$ is estimated by the norm $\|\mathbf{v}\|_{2,2}$, which is less than or equal to $c_{5}\left\|P_{\sigma} \Delta \mathbf{v}\right\|_{2}+c_{6}\|\mathbf{v}\|_{2}$ due to Lemma 3.) Using this inequality in (41), we get

$$
\begin{aligned}
& \|\mathbb{D}(\mathbf{v})\|_{\infty, 2 ;\left(t_{0}, t_{1}\right)}^{2}+\frac{v}{2}\left\|P_{\sigma} \Delta \mathbf{v}\right\|_{2,2 ;\left(t_{0}, t_{1}\right)}^{2} \\
& \leq c_{9}\left\|\mathbb{D}\left(\mathbf{v}\left(\cdot, t_{0}\right)\right)\right\|_{2}^{2} \\
& +c_{10} c_{13}\left\|\left(\zeta_{2}\right)_{+}\right\|_{r, s ;\left(t_{0}, t_{1}\right)}\left(\|\nabla \mathbf{v}\|_{\infty, 2 ;\left(t_{0}, t_{1}\right)}^{2}\right. \\
& \left.\quad+\frac{v}{2}\left\|P_{\sigma} \Delta \mathbf{v}\right\|_{2,2 ;\left(t_{0}, t_{1}\right)}^{2}+c_{14}\right) .
\end{aligned}
$$

Assume that $t_{1}=b_{\gamma}$ and $t_{1}-t_{0}<\xi$, where $\xi$ is so small that $c_{10} c_{13}\left\|\left(\zeta_{2}\right)_{+}\right\|_{r, s ;\left(t^{\prime}, t^{\prime \prime}\right)}<1 / 2$ for any $t^{\prime}, t^{\prime \prime} \in(0, T)$ such that $0 \leq t^{\prime}<t^{\prime \prime} \leq T, t^{\prime \prime}-t^{\prime} \leq \xi$. Then

$$
\begin{gathered}
\|\mathbb{D}(\mathbf{v})\|_{\infty, 2 ;\left(t_{0}, b_{\gamma}\right)}^{2}+\frac{v}{2}\left\|P_{\sigma} \Delta \mathbf{v}\right\|_{2,2 ;\left(t_{0}, b_{\gamma}\right)}^{2} \\
\leq 2 c_{9}\left\|\mathbb{D}\left(\mathbf{v}\left(\cdot, t_{0}\right)\right)\right\|_{2}^{2}+c_{10} c_{13} c_{14} .
\end{gathered}
$$

From this, we observe that $b_{\gamma}$ cannot be an epoch of irregularity of the weak solution $\mathbf{v}$. The proof of Theorem 1 is completed. 


\section{Conflicts of Interest}

The authors declare that they have no conflicts of interest.

\section{Acknowledgments}

The first author has been supported by the Grant Agency of the Czech Republic (Grant no. 17-01747S) and by the Czech Academy of Sciences (RVO 67985840). The second author also appreciates the support of the Czech Academy of Sciences during his stay in Prague.

\section{References}

[1] D. Chae and H.-J. Choe, "Regularity of solutions to the NavierStokes equation," Electronic Journal of Differential Equations, vol. 5, pp. 1-7, 1999.

[2] J. Neustupa and P. Penel, "Anisotropic and geometric criteria for interior regularity of weak solutions to the 3D Navier-Stokes equations," in Mathematical Fluid Mechanics, Recent Results and Open Questions, J. Neustupa and P. Penel, Eds., pp. 237-268, Birkhauser Verlag, Basel-Boston-Berlin, 2001.

[3] M. Pokorný and Y. Zhou, "On the regularity of the solutions of the Navier-Stokes equations via one velocity component," Nonlinearity, vol. 23, no. 5, pp. 1097-1107, 2010.

[4] J. Serrin, "On the interior regularity of weak solutions of the Navier-Stokes equations," Archive for Rational Mechanics and Analysis, vol. 9, pp. 187-195, 1962.

[5] P. Penel and M. Pokorný, "Some new regularity criteria for the Navier-Stokes equations containing gradient of the velocity," Applications of Mathematics, vol. 49, no. 5, pp. 483-493, 2004.

[6] H. Beirão da Veiga, "Concerning the regularity problem for the solutions of the Navier-Stokes equations," Comptes Rendus de $l$ Académie des Sciences - Series I - Mathematics, vol. 321, no. 4, pp. 405-408, 1995.

[7] H. Beirão da Veiga and L. C. Berselli, "On the regularizing effect of the vorticity direction in incompressible viscous flows," Diff. Int. Equations, vol. 15, no. 3, pp. 345-356, 2002.

[8] H. Beirão da Veiga, "Direction of vorticity and regularity up to the boundary: on the Lipschitz-continuous case," Journal of Mathematical Fluid Mechanics, vol. 15, no. 1, pp. 55-63, 2012.

[9] L. C. Berselli and G. P. Galdi, "Regularity criteria involving the pressure for the weak solutions to the Navier-Stokes equations," Proceedings of the American Mathematical Society, vol. 130, no. 12, pp. 3585-3595, 2002.

[10] J. Nečas and J. Neustupa, "New conditions for local regularity of a suitable weak solution to the Navier-Stokes equation," Journal of Mathematical Fluid Mechanics, vol. 4, no. 3, pp. 237-256, 2002.

[11] G. Seregin and V. Šverák, "Navier-Stokes equations with lower bounds on the pressure," Archive for Rational Mechanics and Analysis, vol. 163, no. 1, pp. 65-86, 2002.

[12] J. Neustupa and P. Penel, "Regularity of a weak solution to the Navier-Stokes equation in dependence on eigenvalues and eigenvectors of the rate of deformation tensor," in Progress in Nonlinear Differential Equations and their Applications, J. F. Rodriguez, G. Seregin, and J. M. Urbano, Eds., vol. 61, pp. 197213, Birkhauser, Basel, 2004.

[13] H. J. Choe, "Boundary regularity of weak solutions of the Navier-Stokes equations," Journal of Differential Equations, vol. 149, no. 2, pp. 211-247, 1998.
[14] G. A. Seregin, "Local regularity of suitable weak solutions to the Navier-Stokes equations near the boundary," Journal of Mathematical Fluid Mechanics, vol. 4, no. 1, pp. 1-29, 2002.

[15] L. Caffarelli, R. Kohn, and L. Nirenberg, "Partial regularity of suitable weak solutions of the Navier-Stokes equations," Communications on Pure and Applied Mathematics, vol. 35, no. 6, pp. 771-831, 1982.

[16] S. Gustafson, K. Kang, and T.-P. Tsai, "Regularity criteria for suitable weak solutions of the Navier-Stokes equations near the boundary," Journal of Differential Equations, vol. 226, no. 2, pp. 594-618, 2006.

[17] J. Wolf, "On the boundary regularity of suitable weak solutions to the Navier-Stokes equations," Annali Dell'Universita' Di Ferrara. Sezione VII. Scienze Matematiche, vol. 56, no. 1, pp. 97139, 2010.

[18] G. A. Seregin, T. N. Shilkin, and V. A. Solonnikov, "Partial boundary regularity for the Navier-Stokes equations," Journal of Mathematical Sciences, vol. 132, no. 3, pp. 339-358, 2006.

[19] Z. e. Skalák, "Regularity of weak solutions of the Navier-Stokes equations near the smooth boundary," Electronic Journal of Differential Equations, vol. 45, 11 pages, 2005.

[20] G.-Q. Chen and Z. Qian, "A study of the NAVier-Stokes equations with the kinematic and NAVier boundary conditions," Indiana University Mathematics Journal, vol. 59, no. 2, pp. 721760, 2010.

[21] J. Neustupa and P. Penel, "A weak solution to the navierstokes system with Navier's Boundary condition in a timevarying domain," in Proceedings of the International Conference on Mathematical Fluid Dynamics on the Occasion of Yoshihiro Shibata's 60th Birthday, 2013, pp. 375-400, jpn, March 2013.

[22] P. Kucera and J. Neustupa, "On robustness of a strong solution to the NAVier-Stokes equations with NAVier's boundary conditions in the L3-norm," Nonlinearity, vol. 30, no. 4, pp. 1564-1583, 2017.

[23] G. P. Galdi, "An Introduction to the Navier-Stokes InitialBoundary Value Problem," in Fundamental Directions in Mathematical Fluid Mechanics, G. P. Galdi, J. Heywood, and R. Rannacher, Eds., Advances in Mathematical Fluid Mechanics, pp. 1-98, Birkhauser-Verlag, Basel, 2000.

[24] G. P. Galdi, An Introduction to the Mathematical Theory of the Navier-Stokes Equations, Springer-Verlag, New York-BerlinHeidelberg, 2nd edition, 2011. 


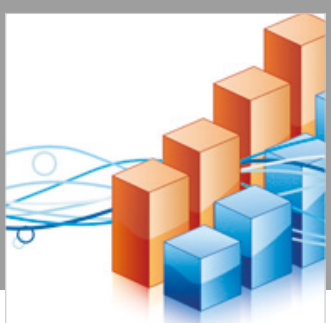

Advances in

Operations Research

\section{-n-m}
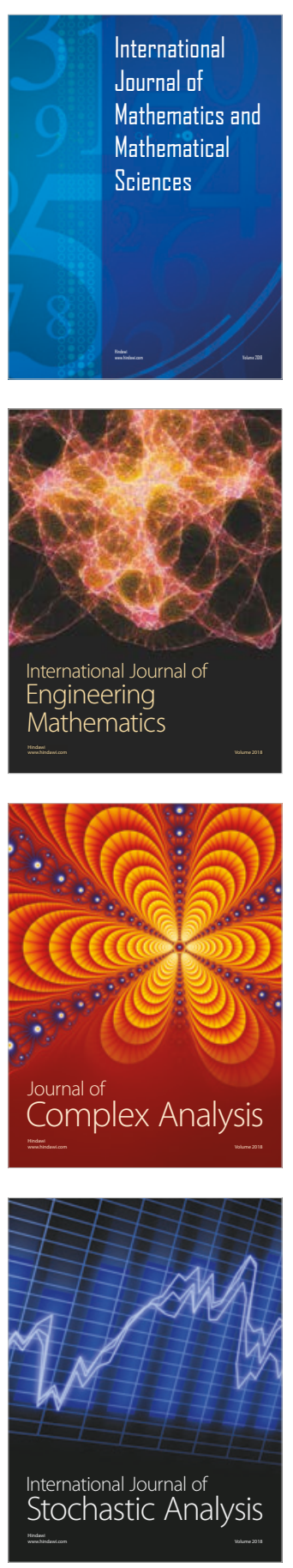
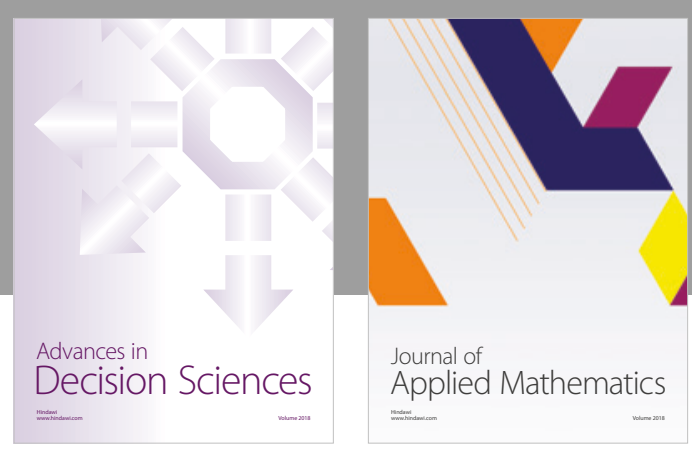

Journal of

Applied Mathematics
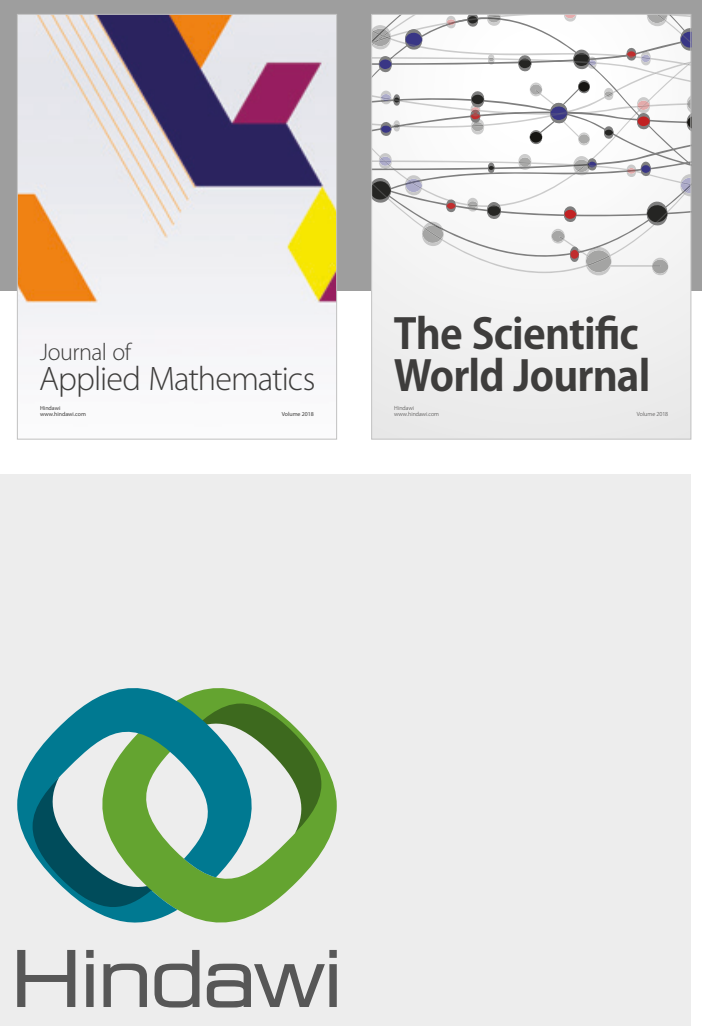

Submit your manuscripts at

www.hindawi.com

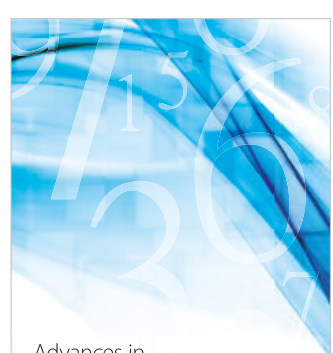

Advances in
Numerical Analysis
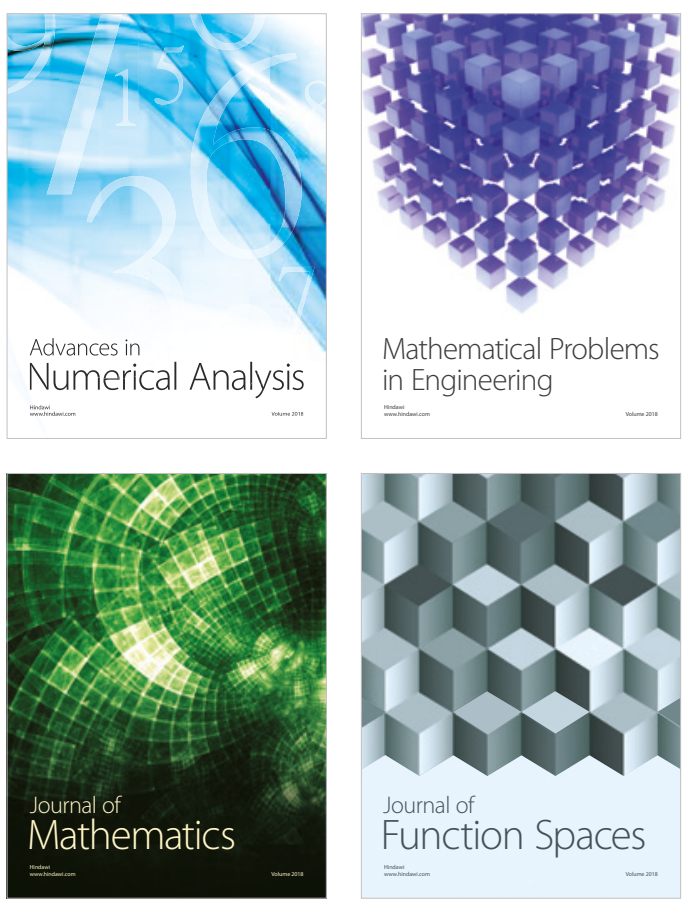

Mathematical Problems in Engineering

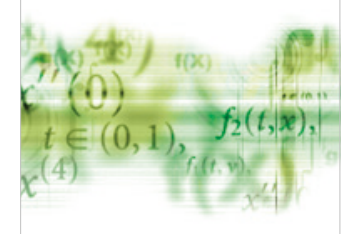

International Journal of

Differential Equations

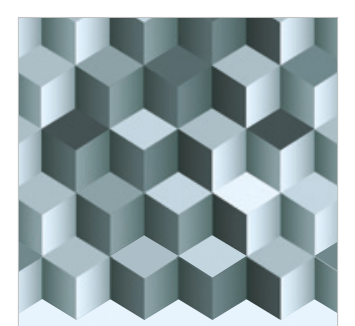

Journal of

Function Spaces

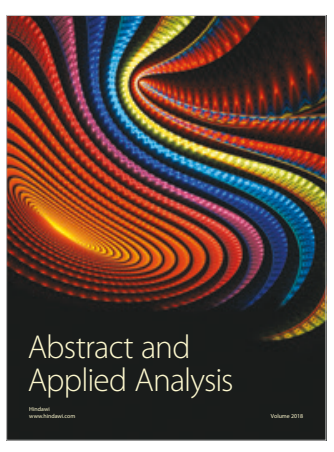

The Scientific

World Journal

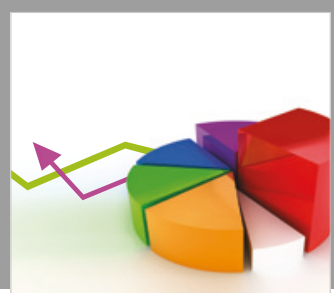

Journal of

Probability and Statistics
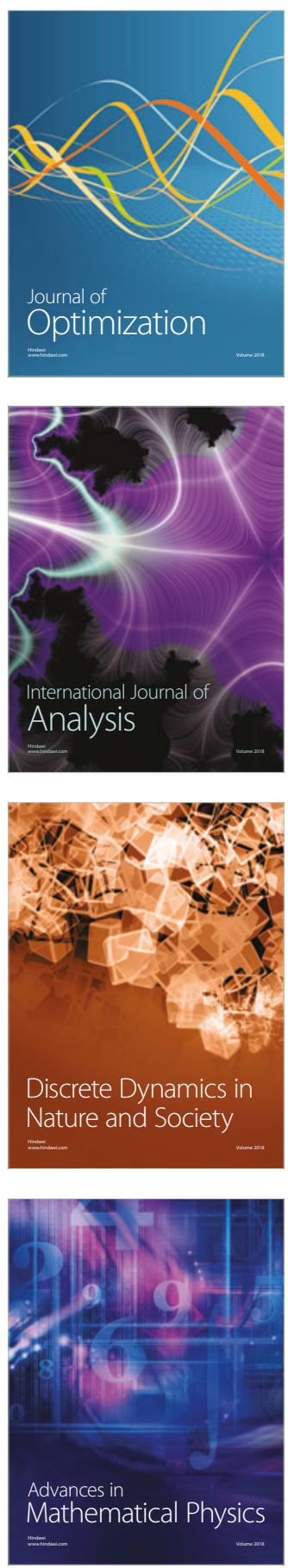